\title{
On the limited increase in validation reliability using high-density genotypes in genomic best linear unbiased prediction: Observations from Fleckvieh cattle
}

\author{
J. Ertl, ${ }^{* 1}$ C. Edel, ${ }^{*}$ R. Emmerling, ${ }^{*}$ H. Pausch,† R. Fries, $†$ and K.-U. Götz ${ }^{*}$ \\ ${ }^{*}$ Institute of Animal Breeding, Bavarian State Research Centre for Agriculture, 85586 Poing, Germany \\ †Chair of Animal Breeding, Technische Universität München, 85354 Freising, Germany
}

\begin{abstract}
This study investigated reliability of genomic predictions using medium-density $(40,089 ; 50 \mathrm{~K})$ or highdensity (HD; 388,951) marker sets. We developed an approximate method to test differences in validation reliability for significance. Model-based reliability and the effect of HD genotypes on inflation of predictions were analyzed additionally. Genomic breeding values were predicted for at least 1,321 validation bulls based on phenotypes and genotypes of at least 5,324 calibration bulls by means of a linear model in milk, fat, and protein yield; somatic cell score; milkability; muscling; udder, feet, and legs score as well as stature. In total, 1,485 bulls were actually HD genotyped and HD genotypes of the other animals were imputed from $50 \mathrm{~K}$ genotypes using FImpute software. Validation reliability was measured as the coefficient of determination of the weighted regression of daughter yield deviations on predicted breeding values divided by the reliability of daughter yield deviations and inflation was evaluated by the slope of this regression. Model-based reliability was calculated from the model. Distributions for validation reliability of $50 \mathrm{~K}$ markers were derived by repeated sampling of 50,000-marker samples from HD to test differences in validation reliability statistically. Additionally, the benefit of HD genotypes in validation reliability was tested by repeated sampling of validation groups and calculation of the difference in validation reliability between $\mathrm{HD}$ and $50 \mathrm{~K}$ genotypes for the sampled groups of bulls. The mean benefit in validation reliability of HD genotypes was 0.015 compared with real $50 \mathrm{~K}$ genotypes and 0.028 compared with $50 \mathrm{~K}$ samples from HD affected by imputation error and was significant for all traits. The model-based reliability was, on average, 0.036 lower and the regression coefficient was 0.036 closer to the expected value with HD genotypes. The observed gain in validation reliability
\end{abstract}

Received March 26, 2013.

Accepted September 16, 2013.

${ }^{1}$ Corresponding author: johann.ertl@lfl.bayern.de with HD genotypes was similar to expectations based on the number of markers and the effective number of segregating chromosome segments. Sampling error in the marker-based relationship coefficients causing overestimation of the model-based reliability was smaller with HD genotypes. Inflation of the genomic predictions was reduced with HD genotypes, accordingly. Similar effects on model-based reliability and inflation, but not on the validation reliability, were obtained by shrinkage estimation of the realized relationship matrix from $50 \mathrm{~K}$ genotypes.

Key words: genomic evaluation, marker density, imputation

\section{INTRODUCTION}

The use of genomic information for the prediction of breeding values is now widespread in dairy cattle breeding all over the world. Genomic breeding values for selection candidates that are predicted with dense marker genotypes are much more reliable than parent averages (VanRaden et al., 2009). Breeding animals are genotyped with high-throughput technologies for thousands of SNP covering the whole genome. Genotyped markers are used as surrogates for unknown QTL in the estimation of the realized relationship matrix. Realized relationships between animals deviate from the expected numerator relationships because of Mendelian sampling during meiosis. In a genomic BLUP (GBLUP) model (VanRaden, 2008), the numerator relationship matrix is replaced by the marker-based relationship matrix. This improves the reliability of breeding values of selection candidates that are predicted based on realized relationships with proven bulls that have phenotypic information. Although currently used assays enable efficient genotyping of more than 50,000 SNP (50K), a high-density (HD) assay was developed for genotyping more than 777,000 SNP, providing genomic information at a higher resolution (Illumina Inc., San Diego, CA). In several investigations, reliabilities of predicted breeding values were compared when breeding values were predicted either from $50 \mathrm{~K}$ or from HD genotypes 
using a GBLUP model, but only minor, if any, gains in validation reliability were observed (Erbe et al., 2012; Su et al., 2012). In this study, we examined the gain in validation reliability from HD genotypes in the Fleckvieh breed, which is known to be genetically more diverse with lower levels of linkage disequilibrium between pairs of markers than Holsteins (Pryce et al., 2011). We inferred distributions for the validation reliability of genomic breeding values predicted with varying $50 \mathrm{~K}$ marker sets to test a potential advantage of $\mathrm{HD}$ genotypes for statistical significance. Furthermore, we analyzed the effect of higher marker density on modelbased reliability and inflation of the predictions. The observed difference in validation reliability with HD genotypes was compared with theoretical expectations.

\section{MATERIALS AND METHODS}

\section{Data}

Genotypes for 21,092 Fleckvieh bulls and candidates were available from the German-Austrian joint genomic evaluation program. The genotypes were generated with Illumina Bovine SNP50 (v1 and v2) Genotyping BeadChips (Illumina Inc.). A total of 1,492 bulls and 2,038 cows were additionally genotyped with an Illumina BovineHD Genotyping BeadChip. The following editing criteria were applied to $50 \mathrm{~K}$ markers: SNP that had a mean call rate below 0.95 or deviated from Hardy-Weinberg equilibrium with $P<10^{-5}$ were excluded from the data set. Genotypes with a minor allele frequency below 0.02 were also discarded. The same editing criteria were applied to HD markers with an adjusted minor allele frequency threshold of 0.005 due to the smaller number of HD genotyped animals. In total, 41,274 50K and 624,892 HD markers passed the quality criteria and were used for imputation. Consistency between genomic and pedigree information was checked by a 2 -step approach. In step 1 , the genotypes of parent-offspring pairs were compared directly and conflicting genotype-configurations were eliminated. In step 2, relationships to genotyped grandsires and (or) within half- and full-sib groups were checked based on marker-based identity-by-descent (IBD) coefficients (Wang, 2007). Conflicting genotypes were either partly or completely set to missing or the sire or the dam was removed from the animal's pedigree following a defined protocol. Imputation of $50 \mathrm{~K}$ to HD genotypes was performed with the program FImpute (Sargolzaei et al., 2011). After imputation, genotypes of 629,028 SNP were available. Although all available genotypes were used for imputation to obtain maximum imputation accuracy by exploiting comprehensive pedigree information, the analysis was based on $50 \mathrm{~K}$ and imputed HD geno- types of 10,240 bulls registered at AI stations and, thus, potentially possessing phenotypic information. Among these 10,240 bulls, 1,492 were actually HD genotyped and HD genotypes of the remaining 8,748 bulls were imputed from $50 \mathrm{~K}$ genotypes; $50 \mathrm{~K}$ and imputed HD genotypes were additionally checked for redundancy. A SNP was considered as redundant when it was in very high linkage disequilibrium $\left(\mathrm{r}^{2}>0.99\right)$ with an adjacent locus. From redundant SNP, the first with regard to UMD3 assembly (http://www.cbcb.umd.edu/research/ bos_taurus_assembly.shtml) position was kept in the data set. For computational reasons, the redundancy check was performed only within chromosomes but not across the whole genome. After exclusion of redundant SNP, 388,951 loci remained in the imputed HD data set and 40,089 remained in the 50K data set.

Phenotypes for the investigation were daughter yield deviations (DYD; VanRaden and Wiggans, 1991) for milk, fat, and protein yield, SCS, stature, muscling, udder, and feet and legs and deregressed proofs (DRP; Garrick et al., 2009) for milkability from the December 2012 German-Austrian routine evaluation. A fraction of 2,265 bulls did not have phenotypes yet.

\section{Genomic Prediction}

Forward predictions were computed using GBLUP (VanRaden, 2008). Bulls born before April 2005 were assigned to the calibration group and the younger bulls served as validation animals. Calibration and validation groups were defined separately for each trait according to the amount of phenotypic information. Only data from validation bulls with phenotypic information equivalent to at least 20 effective daughter contributions (Wilmink and Dommerholt, 1985) in the respective trait were used to validate the genomic predictions. For the different traits, calibration and validation groups consisted of at least 5,324 and 1,321 bulls, respectively. Birth years of analyzed bulls are shown in Figure 1. Direct genomic values (DGV) were predicted for validation bulls based on genotypes and phenotypes of the calibration group. The genomic relationship matrix $\mathbf{G}^{*}$ was calculated following the approach of VanRaden (2008):

$$
\mathbf{G}^{*}=\frac{\mathbf{Z Z}^{\prime}}{2 \sum_{k=1}^{m} p_{k}\left(1-p_{k}\right)},
$$

where $\mathbf{Z}$ is a genotype matrix having a dimension of the number of individuals $(n)$ by the number of loci $(m)$ and is calculated as $\mathbf{Z}=\mathbf{M}-\mathbf{P}$. The elements of $\mathbf{M}$ are -1 and 1 for opposite homozygous genotypes and 0 


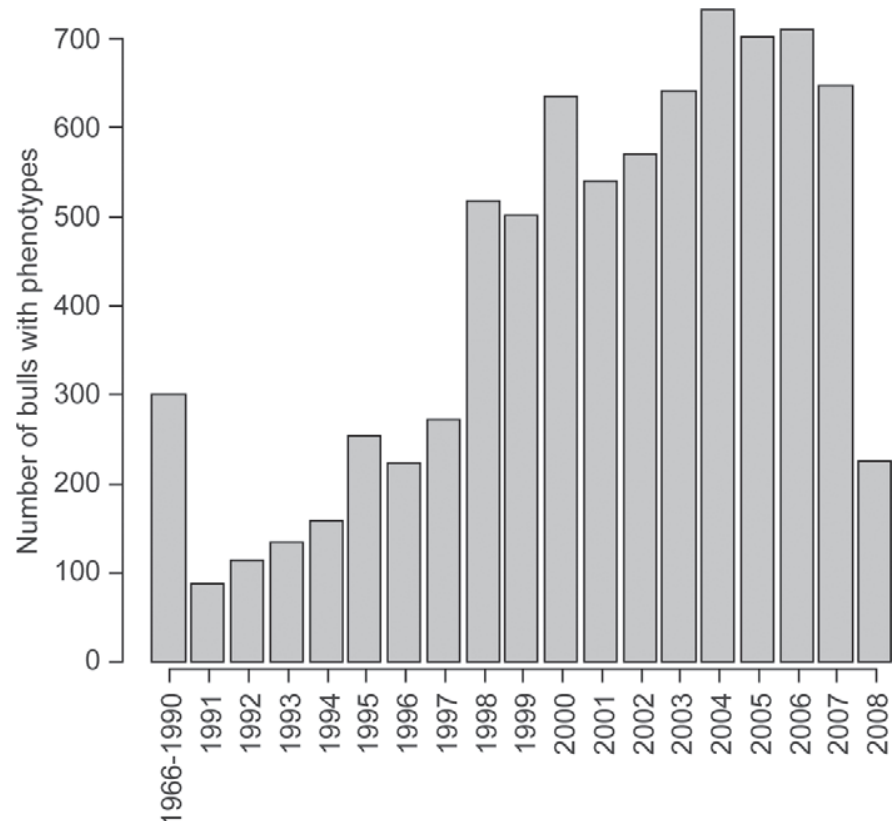

Figure 1. Birth years of bulls with known phenotypes.

for heterozygous genotypes. Column $k$ of the matrix $\mathbf{P}$ is $2\left(p_{k}-0.5\right)$ and $p_{k}$ is the base allele frequency of locus $k$ estimated according to the approach of Gengler et al. (2007); $\mathbf{G}^{*}$ was additionally scaled toward the numerator relationship matrix A (Meuwissen et al., 2011) and finally combined with $\mathbf{A}$ as $\mathbf{G}=0.99 \mathbf{G}^{*}+0.01 \mathbf{A}$ to improve numerical stability.

The following model was applied to predict genomic breeding values $\mathbf{g}$ :

$$
\mathbf{y}=\mu+\mathbf{D g}+\mathbf{e},
$$

where $\mathbf{y}$ is a vector of DRP (milkability) or DYD (other traits) of calibration animals, $\mu$ is the intercept, and $\mathbf{D}$ is a design matrix that relates $\mathbf{y}$ to breeding values; $\mathbf{g}$ is a vector of $\mathrm{DGV}$ for all animals with genotypes in $\mathbf{D}$, including calibration and validation animals. Genomic breeding values were predicted for calibration and validation animals by best linear unbiased prediction (Henderson, 1973):

$$
\hat{\mathbf{g}}=\mathbf{G} \sigma_{a}^{2} \mathbf{D}^{\prime} \mathbf{V}^{-1}\left(\mathbf{y}-\mathbf{1}_{\mathbf{n}} \hat{\mu}\right),
$$

where $\mathbf{1}_{\mathbf{n}}$ is a vector of $1, \hat{\mu}=\left(\mathbf{1}_{\mathbf{n}}{ }^{\prime} \mathbf{V}^{-1} \mathbf{1}_{\mathbf{n}}\right)^{-1} \mathbf{1}_{\mathbf{n}}{ }^{\prime} \mathbf{V}^{-1} \mathbf{y}, \sigma_{a}^{2}$ is the additive genetic variance, and $\mathbf{V}$, the covariance matrix of DYD or DRP, was calculated as

$$
\mathbf{V}=\mathbf{D}\left(\mathbf{G} \sigma_{a}^{2}\right) \mathbf{D}^{\prime}+\mathbf{W} \sigma_{e}^{2},
$$

where $\sigma_{e}^{2}$ is the error variance and $\mathbf{W}$ is a diagonal matrix with reciprocals of the equivalent number of own performances (EOP); EOP are calculated as follows: $\mathrm{EOP}=\frac{\sigma_{e}^{2}}{\sigma_{a}^{2}} \frac{R_{D Y D}^{2}}{1-R_{D Y D}^{2}}$, where $R_{D Y D}^{2}$ is the reliability of the DYD. The parameters $\sigma_{a}^{2}$ and $\sigma_{e}^{2}$ were adopted from the German-Austrian routine evaluation.

The reliability of the prediction as observed in the validation group was measured by means of the coefficient of determination of the weighted regression of DYD on DGV divided by the average reliability of DYD $\left[R^{2}\right.$ (validation)] of validation bulls. We will refer to this reliability as "validation reliability" hereinafter. The model-based reliability $\left[\mathrm{R}^{2}\right.$ (model based)] was computed by direct inversion of the genomic equation system. The weighted regression coefficient of DYD on DGV [b(DYD, DGV)] was calculated to estimate the inflation of the predictions. Inflation means that $\mathrm{b}(\mathrm{DYD}, \mathrm{DGV}$ ) is not equal to its expectation (Mäntysaari et al., 2010). For milkability, DYD were not available and DRP were used instead of DYD to calculate $\mathrm{R}^{2}$ (validation) and $\mathrm{b}(\mathrm{DYD}, \mathrm{DGV})$.

To create an empirical distribution of validation reliabilities of $50 \mathrm{~K}$ predictions, 500 random subsets of 40,089 markers (the number of $50 \mathrm{~K}$ markers in this analysis, designated as $50 \mathrm{~K}$ ) were sampled from the HD markers and used for calibration and prediction of DGV. This kind of comparison is regarded as fair because $50 \mathrm{~K}$ samples are affected by imputation error to the same extent as HD genotypes in contrast to real $50 \mathrm{~K}$ markers that were actually genotyped and thus not affected by imputation error. To obtain samples with a structure similar to the original 50K Illumina BeadChip, the chromosomes were subdivided in bins of $1-\mathrm{Mb}$ length. The number of markers sampled per $1-\mathrm{Mb}$ bin was determined by the number of $50 \mathrm{~K}$ markers observed in this 1-Mb bin. Predictions based on sampled $50 \mathrm{~K}$ markers were performed to obtain an empirical distribution of validation reliability for $50 \mathrm{~K}$ marker sets. For each trait, we calculated the probability that a value from the empirical distribution of $50 \mathrm{~K}$ validation reliabilities exceeds the observed reliability from GBLUP using HD genotypes.

For computational reasons, we performed the sampling of $50 \mathrm{~K}$ markers in all traits with the results from only 1 validation group. However, because the difference in validation reliability between $\mathrm{HD}$ and $50 \mathrm{~K}$ genotypes might also be influenced by the structure of the validation group, we additionally sampled 50 groups of 500 animals with replacement from all potential validation animals. The difference in $\mathrm{R}^{2}$ (validation) between $\mathrm{HD}$ and real $50 \mathrm{~K}$ in each trait was calculated for each validation sample. This difference is the result of the relevant comparison valid in the current situation that all selection candidates are genotyped with $50 \mathrm{~K}$ and 
only a fraction of bulls are HD genotyped. By means of a one-sided $t$-test, we tested the null hypothesis that validation reliability with HD genotypes is not larger than with $50 \mathrm{~K}$ genotypes.

Both Goddard et al. (2011) and Endelman and Jannink (2012) proposed to reduce the sampling error of genomic relationship coefficients, which is basically a function of the number of markers, by shrinking the coefficients toward a target. Goddard et al. (2011) recommended shrinking against the pedigree-based numerator relationship matrix. The shrinkage target of Endelman and Jannink (2012) is a diagonal matrix with elements $1+f$, where $f$ is the mean inbreeding coefficient of the current population. We evaluated the impact of shrinkage estimation of the realized relationship matrix in GBLUP with 50K genotypes, following the approach of Endelman and Jannink (2012), on the validation and model-based reliability as well as on the inflation of DGV and compared the results to the HD results. For this approach, the realized relationship matrix was first calculated as described above using estimates of current allele frequencies. In a second step, the matrix was shrunk toward $1+f$. According to Endelman and Jannink (2012), the genomic relationship matrix (calculated from marker genotypes) $\mathbf{S}=\frac{\mathbf{Z Z}^{\prime}}{m}-\frac{1}{m} \sum_{k=1}^{m} \mathbf{Z}_{\bullet k} \cdot \frac{1}{m} \sum_{k=1}^{m} \mathbf{Z}_{\bullet k}^{\prime}$ has to be shrunk unless the number of markers is much larger than the number of animals to reduce the mean squared error of the genomic relationship coefficients. Shrinkage of $\mathbf{S}$ leads to the realized relationship matrix

$$
\mathbf{G}^{*}=\frac{\delta \frac{1}{n} \sum_{i=1}^{n} \mathbf{S}_{i i} \mathbf{I}+(1-\delta) \mathbf{S}+\frac{1}{m} \sum_{k=1}^{m} \mathbf{Z}_{\bullet k} \cdot \frac{1}{m} \sum_{k=1}^{m} \mathbf{Z}^{\prime} \cdot k}{2 \frac{1}{m} \sum_{k=1}^{m} p_{k}\left(1-p_{k}\right)},
$$

where $\delta$ is the optimal shrinkage intensity ranging from 0 to 1 with the heuristic $\delta \sim \frac{n}{m \cdot C V^{2}}, n$ is the number of genotyped animals, $\mathbf{I}$ is an identity matrix of dimension $n, m$ the number of markers, and $C V$ is the coefficient of variation of the eigenvalues of $\mathbf{S}$. The optimal shrinkage intensity was estimated dependent on the number of markers.

\section{RESULTS}

The validation reliability of genomic breeding values when mainly imputed HD genotypes were used ranged from 0.313 in udder to 0.559 in SCS and was larger than the reliability from $50 \mathrm{~K}$ genotypes for all traits (Table 1). The differences in validation reliability between DGV based on mainly imputed HD and actually genotyped 50K markers, however, were not large and ranged from 0.008 to 0.023 . The mean increase in validation reliability of $\mathrm{HD}$ over all traits was 0.015 . In contrast to the validation reliability, the model-based reliability with HD genotypes was lower than with $50 \mathrm{~K}$ genotypes. The model-based reliability with HD genotypes ranged from 0.547 in feet and legs to 0.662 in milk yield and was 0.626 , on average. The mean model-based reliability for 50K genotypes was 0.662 .

The slopes of the regression of DYD on DGV for HD genotypes ranged from 0.689 in protein yield to 1.024 in milkability and were in all traits closer to the expected value when HD genotypes were used instead of $50 \mathrm{~K}$ genotypes. The mean of $\mathrm{b}(\mathrm{DYD}, \mathrm{DGV})$ over all traits was 0.839 for $\mathrm{HD}$ and 0.803 for $50 \mathrm{~K}$ genotypes. The mean difference in b(DYD, DGV) indicates less inflation of the predictions from HD genotypes. The correlation between $50 \mathrm{~K}$ and HD predictions was between 0.959 and 0.974 for the different traits. This leads to moderate differences in the ranking of the bulls.

Table 1. Validation $\left[\mathrm{R}^{2}\right.$ (validation); adjusted for reliability of daughter yield deviations (DYD)] and modelbased reliability $\left[\mathrm{R}^{2}\right.$ (model based)] and regression coefficients $[\mathrm{b}(\mathrm{DYD}, \mathrm{DGV})$, where $\mathrm{DGV}=$ direct genomic value] of genomic predictions with 50,000-SNP (50K) and high-density (HD) genotypes

\begin{tabular}{lcccccccc}
\hline & \multicolumn{2}{c}{$\mathrm{R}^{2}$ (validation) } & & \multicolumn{2}{c}{$\mathrm{R}^{2}$ (model based) } & & \multicolumn{2}{c}{$\mathrm{b}(\mathrm{DYD}, \mathrm{DGV})$} \\
\cline { 2 - 3 } \cline { 8 - 9 } Trait & $50 \mathrm{~K}$ & $\mathrm{HD}$ & & $50 \mathrm{~K}$ & $\mathrm{HD}$ & & $50 \mathrm{~K}$ & $\mathrm{HD}$ \\
\hline Milk yield & 0.398 & 0.414 & & 0.701 & 0.662 & & 0.700 & 0.739 \\
Fat yield & 0.419 & 0.427 & & 0.696 & 0.657 & & 0.777 & 0.810 \\
Protein yield & 0.378 & 0.392 & & 0.691 & 0.653 & & 0.658 & 0.689 \\
SCS & 0.548 & 0.559 & & 0.679 & 0.643 & & 0.818 & 0.837 \\
Milkability & 0.458 & 0.481 & & 0.685 & 0.648 & & 0.962 & 1.024 \\
Muscling & 0.480 & 0.501 & & 0.627 & 0.594 & & 0.821 & 0.846 \\
Udder & 0.297 & 0.313 & & 0.633 & 0.599 & & 0.845 & 0.886 \\
Feet and legs & 0.315 & 0.330 & & 0.575 & 0.547 & & 0.824 & 0.858 \\
Stature & 0.366 & 0.379 & & 0.669 & 0.632 & & 0.824 & 0.863 \\
Average & 0.407 & 0.422 & & 0.662 & 0.626 & & 0.803 & 0.839 \\
\hline
\end{tabular}


For all analyzed traits, the validation reliability from HD genotypes was clearly larger than the mean of the distribution of $\mathrm{R}^{2}$ (validation) of sampled $50 \mathrm{~K}$ marker sets. As an example, the results from the sampling of 50K markers are shown in Figure 2 for the traits milk yield, SCS, udder, and stature. The dotted line indicates the $95 \%$ quantile of the distribution of validation reliability from sampled $50 \mathrm{~K}$ genotypes. The observed validation reliability from HD genotypes exceeds this 95\% quantile for all traits. The probabilities that a sample of 50K SNP from HD genotypes results in at least the same validation reliability as HD genotypes are given in Table 2 and they are always smaller than $1 \%$ for the different traits.

The validation reliability for sampled $50 \mathrm{~K}$ subsets averaged 0.394 (Table 2) and was 0.013 smaller than that observed from the real $50 \mathrm{~K}$ chip. The difference in validation reliability between $\mathrm{HD}$ and the mean of sampled 50K genotypes ranged from 0.021 to 0.036 , with an average of 0.028 . The sampling of validation animals resulted in statistically significant $(P<0.001)$ differences in validation reliability between $\mathrm{HD}$ and $50 \mathrm{~K}$ genotypes for every trait analyzed.

Applying shrinkage estimation to $50 \mathrm{~K}$ genotypes, had almost no effect on the validation reliability of the genomic prediction. The validation reliability resulting from $50 \mathrm{~K}$ genotypes with shrinkage amounted to 0.409 , on average, and was just 0.002 larger than without shrinkage (Table 3). Model-based reliability from 50K genotypes, however, decreased by 0.026 with shrinkage estimation and approached the model-based reliability from HD genotypes. In the same manner, the regression coefficient b(DYD, DGV) from 50K genotypes with shrinkage approached the result from HD genotypes. On average, it amounted to 0.832 and was 0.029 larger than without shrinkage. The remaining difference to HD genotypes was only 0.007 , on average.

\section{DISCUSSION}

The aim of the present study was to investigate whether a change from $50 \mathrm{~K}$ to $\mathrm{HD}$ genotypes in the German-Austrian routine evaluation would present an immediate benefit that justifies the additional costs. Therefore, we used the standard GBLUP model of the routine evaluation and the same variance components. The use of GBLUP in the genomic analysis is justified, as no clear evidence exists that the infinitesimal model does not apply to the majority of quantitative traits in dairy cattle populations (VanRaden et al., 2009, 2013; Erbe et al., 2012; Su et al., 2012) and especially in the Fleckvieh breed (Gredler et al., 2010; Pausch et al., 2011; Pryce et al., 2011;). Moreover, GBLUP has the practical advantage that genetic base population and variance components do not need to be changed when it is ensured that the genomic relationship matrix has the same scale as the numerator relationship matrix, which should be the case in our study. In this context, the objective of this study was to analyze to what extent the higher marker density improves the estimate of a large genomic relationship matrix and reliability and inflation of genomic predictions in a Fleckvieh population given the GBLUP model.

The use of mainly imputed HD genotypes as compared with $50 \mathrm{~K}$ genotypes resulted in a small gain in validation reliability of 0.015 , on average. Other groups, which compared the reliabilities of genomic breeding values of $50 \mathrm{~K}$ and HD markers predicted with GBLUP, found similar or even smaller differences in reliability between HD and 50K genotypes (Erbe et al., 2012; Su et al., 2012). Gains in validation reliability with HD genotypes were not much larger when nonlinear models were applied (Harris et al., 2011; Erbe et al., 2012; Su et al., 2012; VanRaden et al., 2013). The investigations show that only small, if any, gains in the validation reliability can be obtained with HD genotypes using a GBLUP model. The advantage of 0.015 in validation reliability that we achieved with mainly imputed HD genotypes in our study might not be the true gain that is attainable with HD in the GBLUP model. High-density genotypes were affected by imputation error and this should be taken into account when comparing with results from $50 \mathrm{~K}$ genotypes. Likewise, the HD genotypes in the studies cited above were imputed from $50 \mathrm{~K}$ and thus affected by imputation error. The effect of genotype error on the validation reliability is reported in the Appendix. These results show that the decrease of 0.013 in validation reliability with $50 \mathrm{~K}$ samples compared with real $50 \mathrm{~K}$ genotypes was caused by imputation error in $50 \mathrm{~K}$ samples because introduction of a genotype error equivalent to imputation error in Fleckvieh cattle led to a very similar decay of 0.015 compared with original $50 \mathrm{~K}$ not affected by imputation error.

By means of sampling of 50K marker sets and comparison of the resulting distribution of validation reliability with HD genotypes and sampling of validation animals, we were able to show that the advantage of HD over $50 \mathrm{~K}$ is statistically significant in all 9 analyzed traits. To assess the quality of the $50 \mathrm{~K}$ samples for the estimation of genomic breeding values, we compared the mean of all samples to that of the original 50K set. The difference in validation reliability between sampled and original $50 \mathrm{~K}$ marker sets is likely caused by imputation error affecting imputed HD and sampled 50K genotypes, but not real $50 \mathrm{~K}$ genotypes. The results in Table 1 represent the current situation that all bulls are genotyped with the $50 \mathrm{~K}$ chip and only a fraction of bulls are genotyped with the HD chip. Imputed HD 


\section{Milk yield}

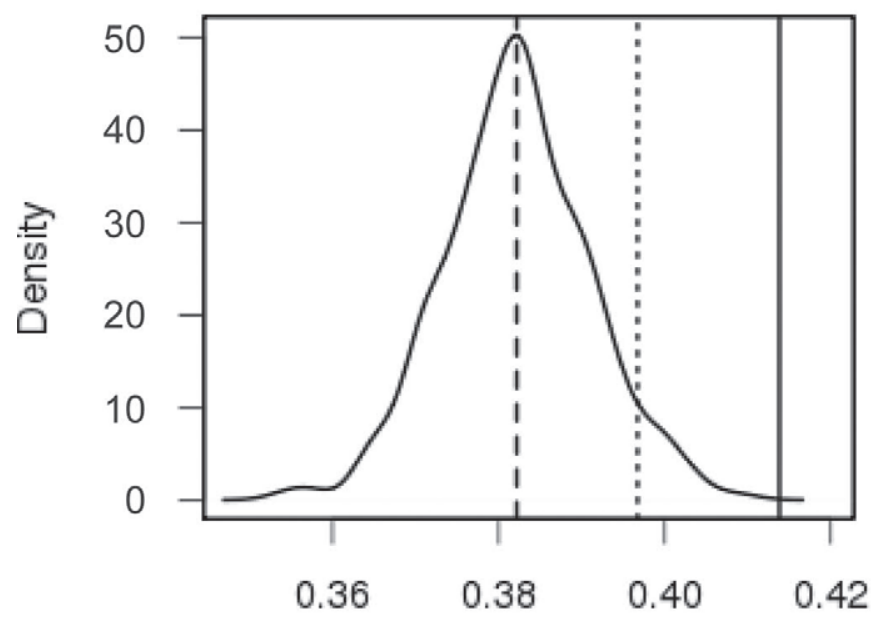

Udder

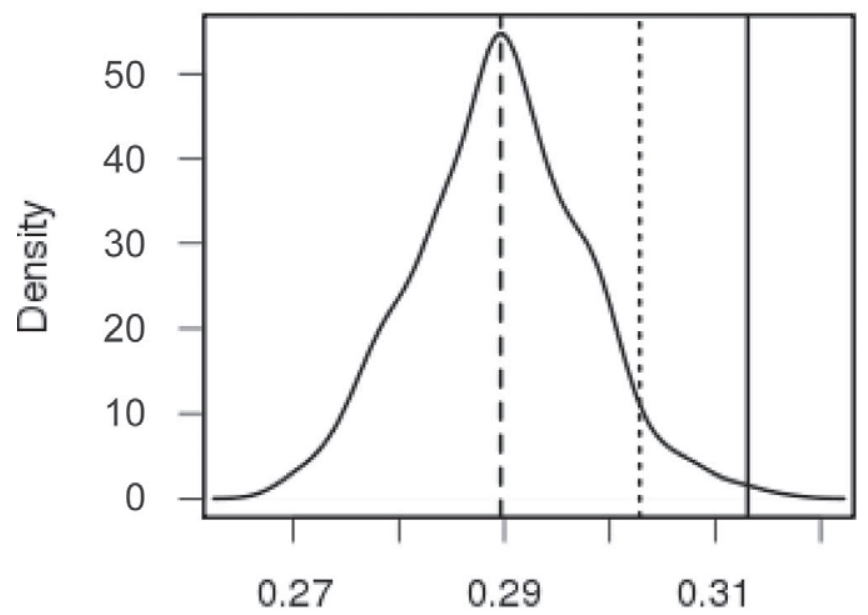

$\mathrm{R}^{2}$ (validation)
Somatic cell score

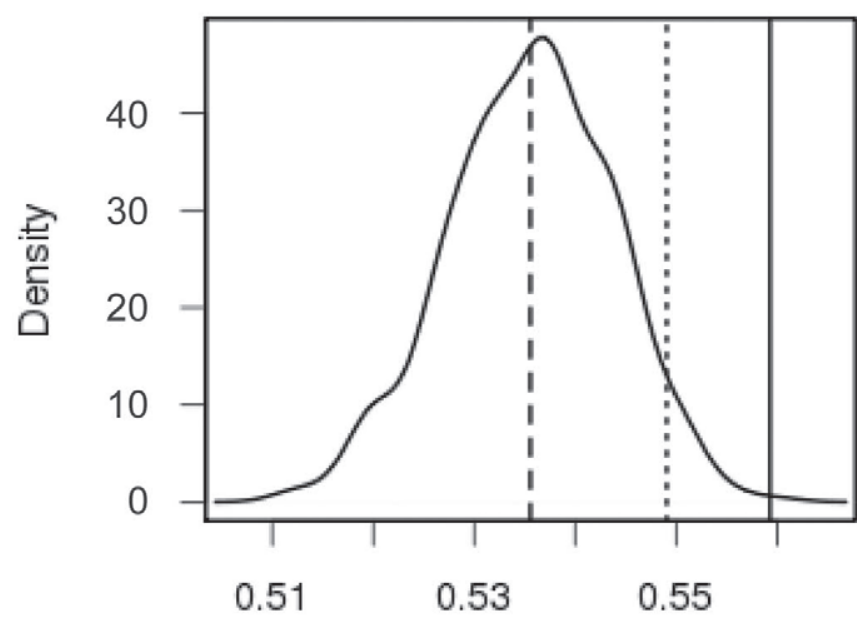

Stature

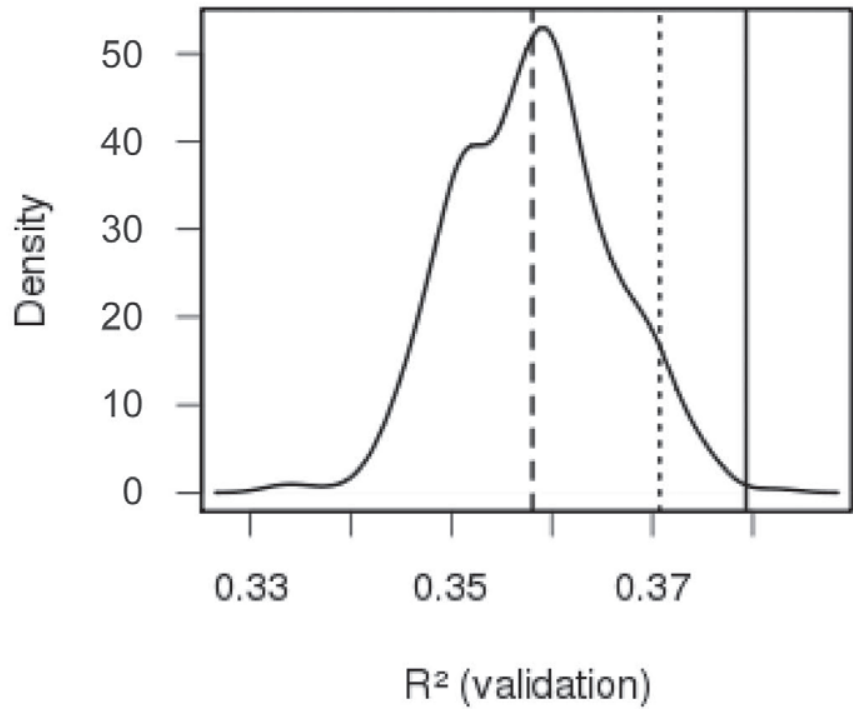

Figure 2. Validation reliability of genomic predictions $\left[\mathrm{R}^{2}\right.$ (validation); adjusted for reliability of daughter yield deviations (DYD) resulting from high-density (HD) markers (solid line) and from sampled 50,000-SNP (50K) subsets from HD (solid curve; dashed line = mean; dotted line $=95 \%$ quantile) in milk yield, SCS, udder, and stature.

genotypes of the remaining bulls are affected by imputation error in contrast to their 50K genotypes. Table 2 summarizes the fair comparison when both marker sets are equally affected by imputation error. These differences apply equally to a setting where all bulls are genotyped with both marker sets or where imputation without error is possible. By means of sampling of validation bulls, we were able to show that the difference between HD and real $50 \mathrm{~K}$ genotypes is significant even under the unfavorable conditions that $50 \mathrm{~K}$ markers are genotyped and HD markers are imputed with some level of error.

The observed small but significant increase in validation reliability with HD genotypes in the GBLUP model should be compared with theoretical expectations. The effective number of chromosome segments segregating in the population (Goddard, 2009; Hayes et al., 2009) is a common measure for haplotype diversity and deter- 
Table 2. Validation reliability [adjusted for reliability of daughter yield deviations (DYD)] of genomic predictions resulting from high-density (HD) markers and from sampled 50,000-SNP (50K) subsets from HD, and $P$-values for the statistical test of the hypothesis that any value from the distribution of $50 \mathrm{~K}$ markers exceeds the validation reliability from HD markers

\begin{tabular}{lccccc}
\hline & \multicolumn{2}{c}{$50 \mathrm{~K}$ subset } & \multicolumn{3}{c}{ Difference } \\
\cline { 2 - 3 } Trait & Mean & $95 \%$ quantile & HD & (HD $-50 \mathrm{~K}$ subset $)$ & $P$-value \\
\hline Milk yield & 0.382 & 0.397 & 0.414 & 0.032 & $1.9 \times 10^{-4}$ \\
Fat yield & 0.393 & 0.408 & 0.427 & 0.034 & $9.4 \times 10^{-5}$ \\
Protein yield & 0.359 & 0.374 & 0.392 & 0.033 & $1.5 \times 10^{-4}$ \\
SCS & 0.536 & 0.549 & 0.559 & 0.023 & $2.0 \times 10^{-3}$ \\
Milkability & 0.445 & 0.458 & 0.481 & 0.036 & $5.6 \times 10^{-6}$ \\
Muscling & 0.474 & 0.487 & 0.501 & 0.027 & $2.4 \times 10^{-4}$ \\
Udder & 0.290 & 0.303 & 0.313 & 0.023 & $1.8 \times 10^{-3}$ \\
Feet and legs & 0.308 & 0.322 & 0.330 & 0.022 & $5.7 \times 10^{-3}$ \\
Stature & 0.358 & 0.371 & 0.379 & 0.021 & $2.9 \times 10^{-3}$ \\
Average & 0.394 & - & 0.422 & 0.028 & - \\
\hline
\end{tabular}

mined by the amount of linkage disequilibrium in the respective population (Goddard et al., 2011). Goddard et al. (2011) derived a theoretical expectation of the reliability of predicted breeding values dependent on the number of calibration animals, mean heritability of the phenotypes, number of markers, and the effective number of chromosome segments segregating in the population. We calculated the expected relative gain from HD markers based on this formula to obtain the expected validation reliability from HD markers. This expectation was consistent with the observed validation reliability from HD markers (Table 4). Contrary to the small gain in validation reliability with HD genotypes, the model-based reliability decreased with the larger number of markers. In a simulation study, Goddard et al. (2011) demonstrated that an increase in the variance of sampling errors of realized relationship coefficients $[\mathrm{V}(\mathrm{E})]$ causes model-based reliability to be overestimated. As $\mathrm{V}(\mathrm{E})$ is reciprocally related to the number of markers (Yang et al., 2010), overestimation of the model-based reliability is reduced with HD geno- types. Using HD genotypes has thus the effect that is intended by the use of shrinkage of estimated genomic relationships (Goddard et al., 2011; Endelman and Jannink, 2012): it reduces the variance of the relationship coefficients and induces accordance of model-based and true reliability.

Endelman and Jannink (2012) showed that the sampling error of the relationship coefficients depends on the ratio of $n$ (number of animals) and $C V^{2}$ (squared coefficient of variation of the eigenvalues of the realized relationship matrix). Notably, with fixed $m$ and $C V$, the sampling error will become larger if the number of genotyped animals increases. Even though the shrinkage target in the method of Endelman and Jannink (2012) is different from that of Goddard et al. (2011), the aim is likewise to reduce the estimation error of realized relationship coefficients. The optimal shrinkage was estimated from our data set for different numbers of markers (depicted in Figure 3). Because the optimal shrinkage intensity decreases hyperbolically with the number of markers, it is negligible for our HD data

Table 3. Validation $\left[\mathrm{R}^{2}\right.$ (validation); adjusted for reliability of daughter yield deviations (DYD)] and modelbased reliability $\left[\mathrm{R}^{2}\right.$ (model based)] and regression coefficients $[\mathrm{b}(\mathrm{DYD}, \mathrm{DGV})$, where $\mathrm{DGV}=$ direct genomic value] of genomic predictions from 50,000-SNP (50K) genotypes with shrinkage estimation (50K shr.) compared with high-density (HD) genotypes

\begin{tabular}{lcccccccc}
\hline & \multicolumn{2}{c}{$\mathrm{R}^{2}$ (validation) } & & \multicolumn{2}{c}{$\mathrm{R}^{2}$ (model based) } & & \multicolumn{2}{c}{$\mathrm{b}(\mathrm{DYD}, \mathrm{DGV})$} \\
\cline { 2 - 3 } Trait & $50 \mathrm{~K}$ shr. & $\mathrm{HD}$ & & $50 \mathrm{~K}$ shr. & $\mathrm{HD}$ & & $50 \mathrm{~K}$ shr. & $\mathrm{HD}$ \\
\hline Milk yield & 0.403 & 0.414 & & 0.672 & 0.662 & & 0.730 & 0.739 \\
Fat yield & 0.422 & 0.427 & & 0.667 & 0.657 & & 0.810 & 0.810 \\
Protein yield & 0.382 & 0.392 & & 0.662 & 0.653 & & 0.684 & 0.689 \\
SCS & 0.547 & 0.559 & & 0.651 & 0.643 & & 0.845 & 0.837 \\
Milkability & 0.466 & 0.481 & & 0.657 & 0.648 & & 1.004 & 1.024 \\
Muscling & 0.480 & 0.501 & & 0.605 & 0.594 & & 0.837 & 0.846 \\
Udder & 0.298 & 0.313 & & 0.610 & 0.599 & & 0.872 & 0.886 \\
Feet and legs & 0.314 & 0.330 & & 0.556 & 0.547 & & 0.848 & 0.858 \\
Stature & 0.370 & 0.379 & & 0.645 & 0.632 & & 0.856 & 0.863 \\
Average & 0.409 & 0.422 & & 0.636 & 0.626 & & 0.832 & 0.839 \\
\hline
\end{tabular}


Table 4. Observed validation reliability [adjusted for reliability of daughter yield deviations (DYD)] with 50,000-SNP (50K) and highdensity (HD) genotypes and expectation of the validation reliability for HD genotypes

\begin{tabular}{lcccc}
\hline & \multicolumn{2}{c}{$50 \mathrm{~K}$} & & \multicolumn{2}{c}{$\mathrm{HD}$} \\
\cline { 2 - 3 } \cline { 5 - 5 } \cline { 5 - 5 } Trait & Observed & & Expected & Observed \\
\hline Milk yield & 0.398 & & 0.407 & 0.414 \\
Fat yield & 0.419 & & 0.428 & 0.427 \\
Protein yield & 0.378 & & 0.386 & 0.392 \\
SCS & 0.548 & & 0.560 & 0.559 \\
Milkability & 0.458 & & 0.468 & 0.481 \\
Muscling & 0.480 & & 0.490 & 0.501 \\
Udder & 0.297 & & 0.303 & 0.313 \\
Feet and legs & 0.315 & & 0.322 & 0.330 \\
Stature & 0.366 & & 0.374 & 0.379 \\
Average & 0.407 & & 0.415 & 0.422 \\
\hline
\end{tabular}

set. From this point of view, the relationships estimated from HD genotypes should reflect the realized relationships at QTL quite well, provided that the distribution of QTL follows approximately the infinitesimal model and that QTL and markers do not differ systematically in their properties.

Shrinkage estimation resulted in a decrease in model-based reliability, whereas validation reliability remained unchanged. Endelman and Jannink (2012) did not detect any increase in prediction accuracy in validation animals with shrinkage estimation either. In contrast to higher marker density, shrinkage estimation does not provide additional information for the prediction of breeding values but ensures that the variance

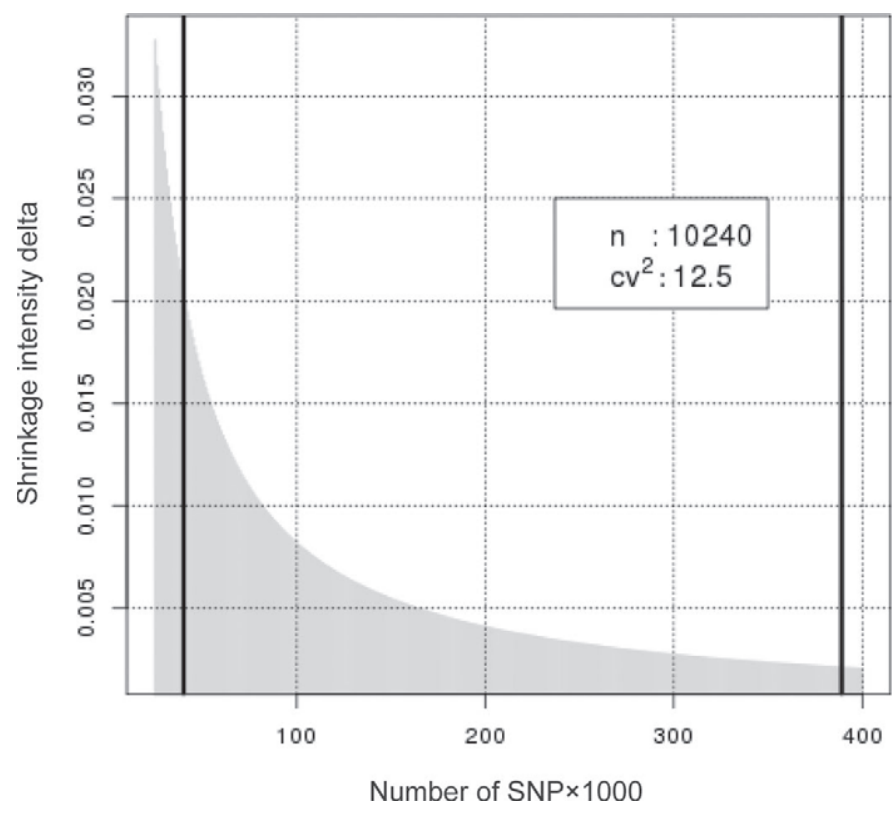

Figure 3. Dependency of the optimal shrinkage intensity on the number of markers in our Fleckvieh data set. of the realized relationship coefficients is not overestimated. Nevertheless, model-based reliabilities in this investigation are still considerably larger than validation reliabilities. Possible reasons for this discrepancy are markers not capturing all of the additive genetic variance, preselection of validation bulls to genotyping and error in validation reliability due to the limited size of the validation group (VanRaden et al., 2009). Preselection of validation bulls to genotyping and errors in the validation reliability due to limited sample size are independent of the number of markers and affect concordance of model-based and validation reliability, irrespective of the marker set. In the German-Austrian genomic routine evaluation, trait-optimized proportions of polygenic variance ranging from 10 to $25 \%$ are included in the analysis to account for genetic variance that is not captured by markers. Therefore, the reported results do not reflect necessarily the situation in the routine evaluation.

Direct genomic values that were predicted with HD genotypes showed less inflation than predictions from $50 \mathrm{~K}$ genotypes. This finding is confirmed by $\mathrm{Su}$ et al. (2012) who predicted genomic values with $50 \mathrm{~K}$ and HD genotypes and reported that the regression coefficient of deregressed proofs on predicted genomic values was larger when HD genotypes were used. As already pointed out, the variance of estimated relationship coefficients is higher with a limited number of markers. The consequence is that the variance of predicted breeding values is inflated. This results in $\mathrm{b}(\mathrm{DYD}, \mathrm{DGV})$ farther from the expectation. With an increasing number of markers, this variance decreases and, consequently, the inflation of the predictions is reduced. However, the inflation of DGV that is still observable with HD probably indicates that even HD does not capture all of the additive genetic variance assumed by the model (Goddard et al., 2011).

\section{CONCLUSIONS}

Prediction of genomic breeding values in the Fleckvieh breed with HD genotypes instead of $50 \mathrm{~K}$ genotypes in a GBLUP model leads to small gains in validation reliability and reduces the inflation of predicted breeding values. Model-based reliabilities that are overestimated with $50 \mathrm{~K}$ genotypes decrease with HD genotypes because the sampling error of the realized relationship matrix is reduced. Similar effects on inflation and model-based reliabilities are obtained by shrinkage estimation of the realized relationship matrix from $50 \mathrm{~K}$ genotypes. However, whereas HD genotypes increase validation reliability, shrinkage estimation only reduces inflation and potentially overestimated modelbased reliability. 


\section{ACKNOWLEDGMENTS}

This research was funded by the German Federal Ministry of Education and Research (Bonn, Germany) within the AgroClustEr "Synbreed-Synergistic plant and animal breeding" (grant no. $0315628 \mathrm{H}$ ). We thank M. Sargolzaei for providing his imputation software FImpute. The Förderverein Biotechnologieforschung e.V. (Bonn, Germany) and the organizations contributing to the German-Austrian pool of Fleckvieh genotypes are gratefully acknowledged for permitting the use of $50 \mathrm{~K}$ genotypes. The manuscript has benefited from critical comments of 2 anonymous reviewers.

\section{REFERENCES}

Chen, J., Z. Liu, F. Reinhardt, and R. Reents. 2011. Reliability of genomic prediction using imputed genotypes for German Holsteins: Illumina $3 \mathrm{~K}$ to $54 \mathrm{~K}$ bovine chip. The 2011 Interbull Open Meeting, Stavanger, Norway. Interbull, Uppsala, Sweden.

Dassonneville, R., R. F. Brøndum, T. Druet, S. Fritz, F. Guillaume, B. Guldbrandtsen, M. S. Lund, V. Ducrocq, and G. Su. 2011. Effect of imputing markers from a low-density chip on the reliability of genomic breeding values in Holstein populations. J. Dairy Sci. 94:3679-3686. http://dx.doi.org/10.3168/jds.2011-4299.

Endelman, J. B., and J.-L. Jannink. 2012. Shrinkage estimation of the realized relationship matrix. G3 (Bethesda) 2:1405-1413. http:// dx.doi.org/10.1534/g3.112.004259.

Erbe, M., B. J. Hayes, L. K. Matukumalli, S. Goswami, P. J. Bowman, C. M. Reich, B. A. Mason, and M. E. Goddard. 2012. Improving accuracy of genomic predictions within and between dairy cattle breeds with imputed high-density single nucleotide polymorphism panels. J. Dairy Sci. 95:4114-4129. http://dx.doi.org/10.3168/ jds.2011-5019.

Garrick, D. J., J. F. Taylor, and R. L. Fernando. 2009. Deregressing estimated breeding values and weighting information for genomic analyses. Genet. Sel. Evol. 41:55 http://dx.doi.org/10.1186/12979686-41-55.

Gengler, N., P. Mayeres, and M. Szydlowski. 2007. A simple method to approximate gene content in large pedigree populations: Application to the myostatin gene in dual-purpose Belgian Blue cattle. Animal 1:21-28. http://dx.doi.org/10.1017/S1751731107392628.

Goddard, M. 2009. Genomic selection: Prediction of accuracy and maximisation of long term response. Genetica 136:245-257. http://dx.doi.org/10.1007/s10709-008-9308-0.

Goddard, M. E., B. J. Hayes, and T. H. E. Meuwissen. 2011. Using the genomic relationship matrix to predict the accuracy of genomic selection. J. Anim. Breed. Genet. 128:409-421. http://dx.doi. org/10.1111/j.1439-0388.2011.00964.x.

Gredler, B., H. Schwarzenbacher, C. Egger-Danner, C. Fuerst, R. Emmerling, and J. Sölkner. 2010. Accuracy of genomic selection in dual purpose Fleckvieh cattle using three types of methods and phenotypes. Proc. 9th World Congr. Genet. Appl. Livest. Prod., Leipzig, Germany. Gesellschaft für Tierzuchtwissenschaften e.V., Gießen, Germany.

Harris, B. L., F. E. Creagh, A. M. Winkelman, and D. L. Johnson. 2011. Experiences with the Illumina high density Bovine BeadChip. Interbull Bull. 44:3-7.

Hayes, B. J., P. J. Bowman, A. J. Chamberlain, and M. E. Goddard. 2009. Invited review: Genomic selection in dairy cattle: Progress and challenges. J. Dairy Sci. 92:433-443. http://dx.doi. org/10.3168/jds.2008-1646.

Henderson, C. R. 1973. Sire evaluation and genetic trends. Pages 10-412 in Proceedings of the Animal Breeding and Genetics Symposium in Honor of Dr. J. L. Lush. American Society of Animal Science and American Dairy Science Association, Champaign, IL.
Mäntysaari, E., Z. Liu, and P. VanRaden. 2010. Interbull validation test for genomic evaluations. Interbull Bull. 41:17-22.

Meuwissen, T. H. E., T. Luan, and J. A. Woolliams. 2011. The unified approach to the use of genomic and pedigree information in genomic evaluations revisited. J. Anim. Breed. Genet. 128:429-439. http://dx.doi.org/10.1111/j.1439-0388.2011.00966.x.

Pausch, H., K. Flisikowski, S. Jung, R. Emmerling, C. Edel, K.-U. Götz, and R. Fries. 2011. Genome-wide association study identifies two major loci affecting calving ease and growth-related traits in cattle. Genetics 187:289-297. http://dx.doi.org/10.1534/genetics.110.124057.

Pryce, J. E., B. Gredler, S. Bolormaa, P. J. Bowman, C. Egger-Danner, C. Fuerst, R. Emmerling, J. Sölkner, M. E. Goddard, and B. J. Hayes. 2011. Short communication: Genomic selection using a multi-breed, across-country reference population. J. Dairy Sci. 94:2625-2630. http://dx.doi.org/10.3168/jds.2010-3719.

Sargolzaei, M., J. P. Chesnais, and F. S. Schenkel. 2011. FImputeAn efficient imputation algorithm for dairy cattle populations. J. Dairy Sci. 94(E-Suppl. 1):421. (Abstr.)

Su, G., R. F. Brøndum, P. Ma, B. Guldbrandtsen, G. P. Aamand, and M. S. Lund. 2012. Comparison of genomic predictions using medium-density $(\sim 54,000)$ and high-density $(\sim 777,000)$ single nucleotide polymorphism marker panels in Nordic Holstein and Red Dairy Cattle populations. J. Dairy Sci. 95:4657-4665. http:// dx.doi.org/10.3168/jds.2012-5379.

VanRaden, P. M. 2008. Efficient methods to compute genomic predictions. J. Dairy Sci. 91:4414-4423. http://dx.doi.org/10.3168/ jds.2007-0980.

VanRaden, P. M., D. J. Null, M. Sargolzaei, G. R. Wiggans, M. E. Tooker, J. B. Cole, T. S. Sonstegard, E. E. Connor, M. Winters, J. B. C. H. M. van Kaam, A. Valentini, B. J. Van Doormaal, M. A. Faust, and G. A. Doak. 2013. Genomic imputation and evaluation using high-density Holstein genotypes. J. Dairy Sci. 96:668-678. http://dx.doi.org/10.3168/jds.2012-5702.

VanRaden, P. M., C. P. Van Tassell, C. R. Wiggans, T. S. Sonstegard, R. D. Schnabel, J. F. Taylor, and F. S. Schenkel. 2009. Invited review: Reliability of genomic predictions for North American Holstein bulls. J. Dairy Sci. 92:16-24. http://dx.doi.org/10.3168/ jds.2008-1514.

VanRaden, P. M., and G. R. Wiggans. 1991. Derivation, calculation, and use of national animal model information. J. Dairy Sci. 74:27372746. http://dx.doi.org/10.3168/jds.S0022-0302(91)78453-1.

Wang, J. 2007. Triadic IBD coefficients and applications to estimating pairwise relatedness. Genet. Res. 89:135-153. http://dx.doi. org/10.1017/S0016672307008798.

Wilmink, J. B. M., and J. Dommerholt. 1985. Approximate reliability of best linear unbiased prediction in models with and without relationships. J. Dairy Sci. 68:946-952.

Yang, J., B. Benyamin, B. P. McEvoy, S. Gordon, A. K. Henders, D. R. Nyholt, P. A. Madden, A. C. Heath, N. G. Martin, G. W. Montgomery, M. E. Goddard, and P. M. Visscher. 2010. Common SNPs explain a large proportion of the heritability for human height. Nat. Genet. 42:565-569. http://dx.doi.org/10.1038/ng.608.

\section{APPENDIX}

We found that shrinkage of the identity-by-state (IBS) matrix results in lower model-based reliability, whereas validation reliability remains constant. To assess the effect of wrongly imputed genotypes on the validation reliability, we introduced different degrees of error into the 50K genotypes. For each animal, a proportion of genotypes was drawn at random and substituted by false genotypes. When the original genotype was heterozygous, it was replaced by one of the homozygotes with equal probability. Homozygous geno- 
Table 5. Validation reliability [adjusted for reliability of daughter yield deviations (DYD)] from original 50,000-SNP (50K) genotypes, $50 \mathrm{~K}$ genotypes with $1.5 \%$ homogeneous error, and sampled $50 \mathrm{~K}$ genotypes from high density (HD)

\begin{tabular}{lccc}
\hline Trait & $50 \mathrm{~K}$ & $\begin{array}{c}50 \mathrm{~K} \text { with } \\
1.5 \% \text { error }\end{array}$ & $\begin{array}{c}50 \mathrm{~K} \\
\text { subset }\end{array}$ \\
\hline Milk yield & 0.398 & 0.384 & 0.382 \\
Fat yield & 0.419 & 0.399 & 0.393 \\
Protein yield & 0.378 & 0.362 & 0.359 \\
SCS & 0.548 & 0.527 & 0.536 \\
Milkability & 0.458 & 0.452 & 0.445 \\
Muscling & 0.480 & 0.466 & 0.474 \\
Udder & 0.297 & 0.286 & 0.290 \\
Feet and legs & 0.315 & 0.298 & 0.308 \\
Stature & 0.366 & 0.355 & 0.358 \\
Average & 0.407 & 0.392 & 0.394 \\
\hline
\end{tabular}

types were replaced by either the heterozygote or the alternative homozygote with equal probability. Thus, $50 \mathrm{~K}$ data sets were created with $0.5,1.0,1.5,2.0,2.5$, and $3.0 \%$ false genotypes and used for the prediction of genomic breeding values. The validation reliability was calculated as the coefficient of determination of the regression of DYD on DGV of validation animals divided by the reliability of DYD of validation animals. For each error percentage, 30 replicates were performed to obtain a reliable estimate of $\mathrm{R}^{2}$ (validation).

The effect of erroneous genotypes on the validation reliability is presented in Figure 4 for different error rates. The validation reliability decreased almost linearly with increasing error percentage. The extent of the decrease differed between the traits. Fat and protein yield were most and milkability was least affected by genotype errors. Validation reliabilities are reported for $1.5 \%$ false genotypes in Table 5 and compared with mean validation reliability from $50 \mathrm{~K}$ samples and validation reliability from the $50 \mathrm{~K}$ chip. Validation

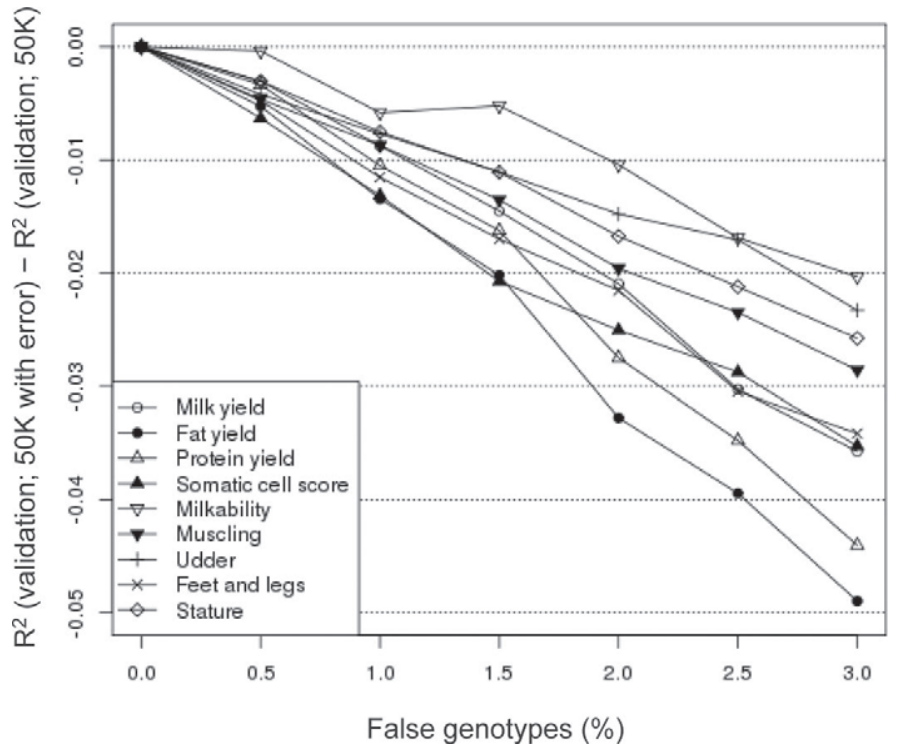

Figure 4. Decay in validation reliability [adjusted for reliability of daughter yield deviations (DYD)] for different percentages of false genotypes. $50 \mathrm{~K}=50,000 \mathrm{SNP}$.

reliability from $50 \mathrm{~K}$ genotypes with $1.5 \%$ errors was, on average, 0.015 lower than validation reliability from original $50 \mathrm{~K}$ genotypes. The decay in validation reliability with $1.5 \%$ error was very similar to the decrease with sampled $50 \mathrm{~K}$ subsets from HD genotypes. This indicates that the imputation error was approximately equivalent to a homogeneous genotype error of $1.5 \%$. Analysis of imputation from 50K to HD with FImpute in Fleckvieh resulted in 1.6\% genotype error. Similar reductions of validation reliability with imputation error were reported by Chen et al. (2011) and Dassonneville et al. (2011). 